\title{
An easy way to put the spleen into the bag
}

\author{
Murat Cakir, Ahmet Tekin, Adil Kartal, Fatma Betul Tuncer \\ Department of General Surgery, Meram Medical Faculty, Necmettin Erbakan University, Konya, Turkey
}

Videosurgery Miniinv 2013; 8 (3): 241-243

DOI: $10.5114 /$ wiitm.2011.33612

\begin{abstract}
Introduction: Splenectomy is a therapeutic and diagnostic procedure used in a wide range of situations. Laparoscopic splenectomy has become the gold standard in some hematological diseases. The laparoscopically removed spleen is placed into a surgical bag, a step which is sometimes the most time-consuming part of the operation.

Aim: To present the method that we employed in laparoscopic splenectomy to place the specimen into the bag and extract it in an easier and simpler way.

Material and methods: The proximal part of the splenorenal ligament is left undivided in the size of one LigaSure cut length to use as a stalk while placing the spleen into the surgical bag. The bag is advanced from the inferior pole of the spleen toward the superior pole. Only keeping the bag open is sufficient to place the spleen into the bag.

Results: Recently, me started to put the spleen into the bag easily before cutting upper attachment of the spleen laparoscopically. So far we applied this procedure in more than eleven cases without complication.

Conclusions: Splenectomy is now the gold standard in the treatment of hematologic diseases that are resistant to medical treatment or that are not amenable to medical treatment because of its complications. Through our experience, the method that we describe here easily overcomes one of the most unpleasant parts of laparoscopic splenectomy.
\end{abstract}

Key words: laparoscopy, splenectomy, bag.

\section{Introduction}

Splenectomy is a therapeutic and diagnostic procedure used in a wide range of situations. Compared with the open technique, the main advantages of laparoscopic splenectomy (LS) include less pain, faster recovery of intestinal motility, shorter hospitalization period and better cosmesis [1-5]. Advances in surgical techniques and accumulating expertise in laparoscopic surgery have led to rapid improvement in the field of laparoscopic splenectomy compared to that in conventional surgery. Laparoscopic splenectomy has become the gold standard in some of the hematological diseases in which splenectomy has been indicated.
Laparoscopic splenectomy was first described by Cadiere in 1993 [6, 7]. Some minor modifications such as positioning of the patient, diameters of trocars, dissecting instruments and extraction of the spleen occurred in different parts of the procedure after its original description. The most important change in the technique of LS concerns extraction of the spleen.

Laparoscopic removal of the spleen includes mobilization of the spleen by dividing the splenocolic, lienophrenic and splenorenal ligaments, ligation of the short gastric vessels, and then dissection and ligation of the hilar vessels with clips, staplers or thermal energy devices. Once all the attachments of the spleen are divided, the spleen is placed into an 


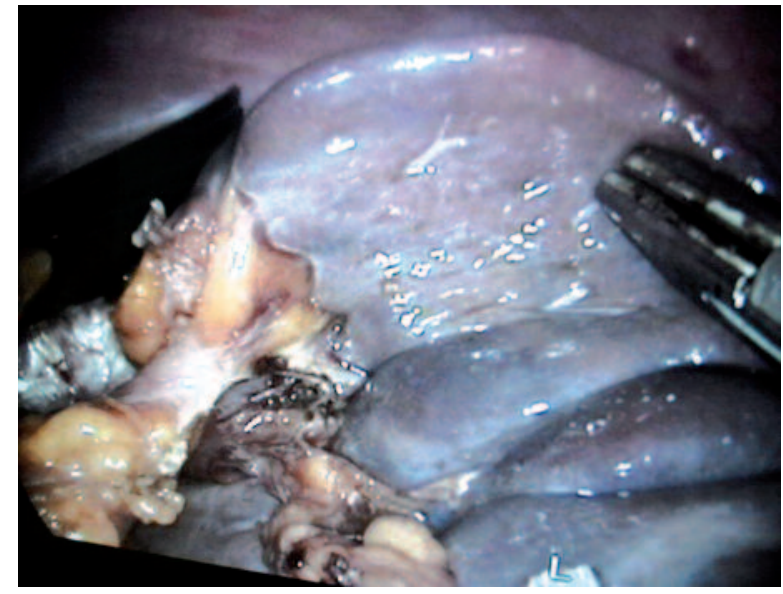

Photo 1. Proximal splenorenal ligament left intact

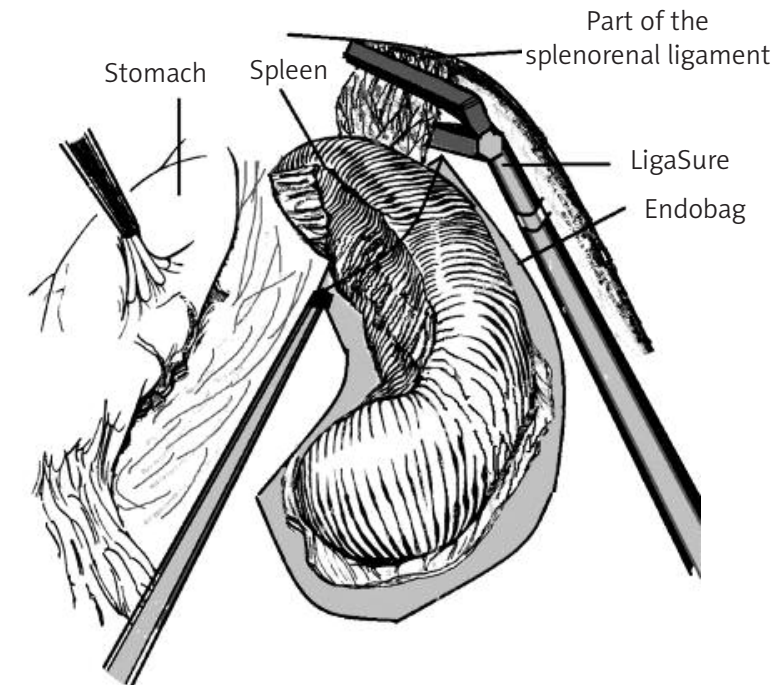

Figure 1. The spleen to be in the bag. After cutting the last part of the splenorenal ligament

A bag is introduced from the port and is opened in the abdominal cavity. The bag is advanced from the inferior pole of the spleen toward the superior pole. Only keeping the bag open is sufficient to place the spleen into the bag (Figure 1). This technique does not allow slippage or displacement of the spleen as happens in other methods where all the attachments of the spleen are freed. The spleen, even if it rotates, does not change its place. Then, the spleen can be easily placed into the bag through its single attachment. After almost the whole spleen is placed into the bag, the proximal part of the splenorenal ligament that was left to fix the spleen or to use as a stalk is divided by use of the LigaSure. Thus, the whole spleen can be placed into the endobag by its own gravity.

\section{Discussion}

The exteriorization of the spleen that has been freed from all of its attachments is a challenging part of LS. Through our experience, the method that we have described above easily overcomes one of the most unpleasant parts of LS. This technique is an easy way to put the spleen into the bag. It can be learned easily and quickly.

\section{References}

1. Balagué C, Targarona EM, Cerdán G, et al. Long-term outcome after laparoscopic splenectomy related to hematologic diagnosis. Surg Endosc 2004; 18: 1283-7. 
2. Durakbasa CU, Timur C, Sehiralti V, et al. Pediatric splenectomy for hematological diseases: outcome analysis. Pediatr Surg Int 2006; 22: 635-9.

3. Velanovich V, Shurafa MS. Clinical and quality of life outcomes of laparoscopic and open splenectomy for hematological diseases. Eur J Surg 2001; 167: 23-8.

4. Stefaniak TJ, Adamczyk K, Walerzak A, et al. Body image is an important augmentation to quality of life in evaluating the results of laparoscopic versus classic surgery. Videosurgery Miniinv 2010; 5: 146-51.

5. Dzielicki J, Grabowski A, Korlacki W. Optimizing the technique of laparoscopic splenectomy in children. Videosurgery Miniinv 2010; 5: 19-26.

6. Cadiere GB, Verroken R, Himpens J, et al. Operative strategy in laparoscopic splenectomy. J Am Coll Surg 1994; 179: 668-72.

7. Park A, Gagner M, Pomp A. The lateral approach to laparoscopic splenectomy. Am J Surg 1997; 173: 126-30.

Received: 19.05.2012, accepted: 3.10.2012. 